\title{
Analysis of Positive and Negative Emotions based on EEG Signal
}

\author{
Juan Li ${ }^{\text {a }}$, Guozhong Liu ${ }^{\mathrm{b}}$ and Jie Gao ${ }^{\mathrm{c}}$ \\ School of Instrument Science and Opto-electronics Engineering, Beijing Information Science \& \\ Technology University, Beijing 100192, China
}

a359199235@qq.com, bliuguozhong@bistu.edu.cn, c2604120131@qq.com

Keywords: EEG; SVM; Emotion; Wavelet Entropy; CSP.

\begin{abstract}
The use of computer technology for emotion recognition is the key to achieve high-level human-computer interaction. Aiming at the research of emotion recognition and classification based on EEG signals, emotional stimulus was designed with positive and negative emotions. The emotion feature extraction based on algorithm of Common spatial pattern (CSP) after signal denoising. The emotion classification algorithm based on the support vector machine (SVM), and the finally classification accuracy can achieve $92 \%$. Lastly, we analyze the complexity of EEG data in the by using the wavelet entropy algorithm. It found that wavelet entropy value of the negative emotion state is lower than the positive state. It shows that the brain is nervous and regularity of the brain is stronger when it is negative. In the positive mood, however, the brain is relatively relaxed and the regularity becomes weak. The study realizes the visualization of human emotion, which provides great value for the study of depression and those who can be easily influenced by emotion for heavy stress.
\end{abstract}

\section{Introduction}

Emotion, as a function of human brain, is an important part of human intelligence, which influences learning, memory and decision-making to different extent, guarantees the survival and the adaptation of the organism, and is the key factors of personality traits and mental pathology. It is the key technology to realize the advanced human-computer interaction by using computer technology to carry on the emotion recognition. It has important significance for the realization of humancomputer interaction, human -computer interface and intelligent computer. Emotion has become a hot topic that the study of emotion recognition based on EEG in the field of neural engineering and biomedical engineering. Therefore, the analysis and recognition of emotion is an important interdiscipline research topic in the fields of neuroscience, psychology, cognitive science, computer science, artificial intelligence and so on.

Emotional research has a long history. The researchers put forward different opinions and theories in the field of emotion research. They reveal the emergence and development of emotion from different angles. At present, there are two main theories of emotion. One kind is the basic emotion theory which is represented by Ekman [3]. Including the positive emotions (interest, pleasure and other basic) as well as the basic negative emotions (sadness, anger, tension, etc.) The other is the theory of the dimension of emotion represented by Russell [4-5] et al. It is composed of two dimensions: pleasure and arousal.

In this paper, we adopt the first point of view. To observe the difference of EEG characteristics between positive and negative emotions. The objective is to show the different features of the EEG signals. To infer the different human emotions.

\section{Design of experimental system and scheme}

BCI system includes the subject, PC, Visual stimulus interface, Brain electrical signal amplifier and brain electrical signal real-time monitoring. The overall BCI system chart is shown in figure 1. 


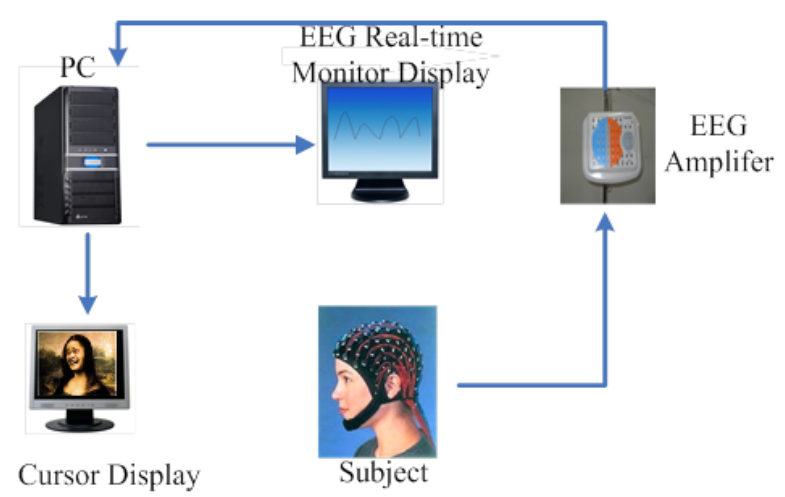

Figure 1. System block diagram of experiment

In this study, we adopted NT9200 digital electroencephalograph as our experimental instrument, with sampling rate of $1000 \mathrm{~Hz}, 32$ channels. When receiving the paper, we assume that the corresponding authors grant us the copyright to use. For this system chart, explanation is as follows: in the process of the experiment, ensure that the subject is in the best state of mind, avoids overly nervous or tired.

After the start of the experiment, the center of the screen appears "ready" message box, according to the prompt, the subjects make the arms and the shoulders relaxed, the eyes straight ahead center screen or natural closed, and make the brain in a relaxed state. After four seconds to relax, the subjects enter the state of the task, with the center of the screen showing the stimulus picture of emotion, the subjects carry out the experiment according to the category of the mood by the performance of the animation and the experimenter's prompt. The experiment selected 20 right hands as the subjects whose ages are between 22-27 years old, where men and women accounted for half.

The stimulus phase of the experimental is disorder played by the positive and the negative animations whose number is a total of one hundred. The picture is selected by the reviewing results of 52 Teachers and students in the school together to, which fit in with the Chinese people's emotional expression. It has a $3 \mathrm{~S}$ blank time between the two animations, without any stimulation, It is for the preparation of the next stimulus.

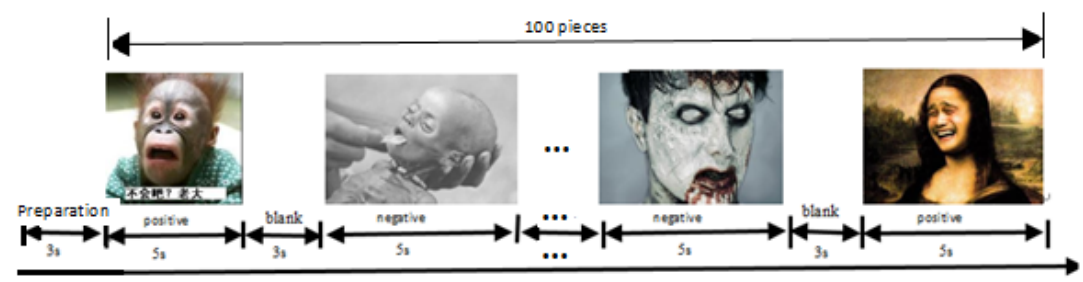

Figure 2. Experimental stimulus sequence diagram

\section{Data preprocessing.}

After signal denoising though spatial filter,we need to extract the features of the signal by the algorithm of Common spatial pattern (CSP).CSP algorithm is proposed by Fukunage [6]. CSP algorithm is to find the space projection, makes the same class in distance as small as possible, the different classes in distance as large as possible, lay a good foundation for subsequent classification algorithm.

EEG data is divided into training set and testing set, spatial filter is trained by the training set, and then then extract the feature of test data through the spatial filter. Thus the processing of the training EEG data is key to construct the spatial filter. EEG data is shown as $\mathrm{N} \times \mathrm{T} \times \mathrm{M}$ dimensional matrix $\mathrm{X}$. Where $\mathrm{N}$ is the number of channels, $\mathrm{T}$ is sampling points of each channel and is the number of experiments. We use CSP algorithm for feature extraction of training data set in this paper, the two kinds of sample matrix are train_positive and train_negtive after data preprocessing. The format of data is $32 \times 2000 \times 50$. 

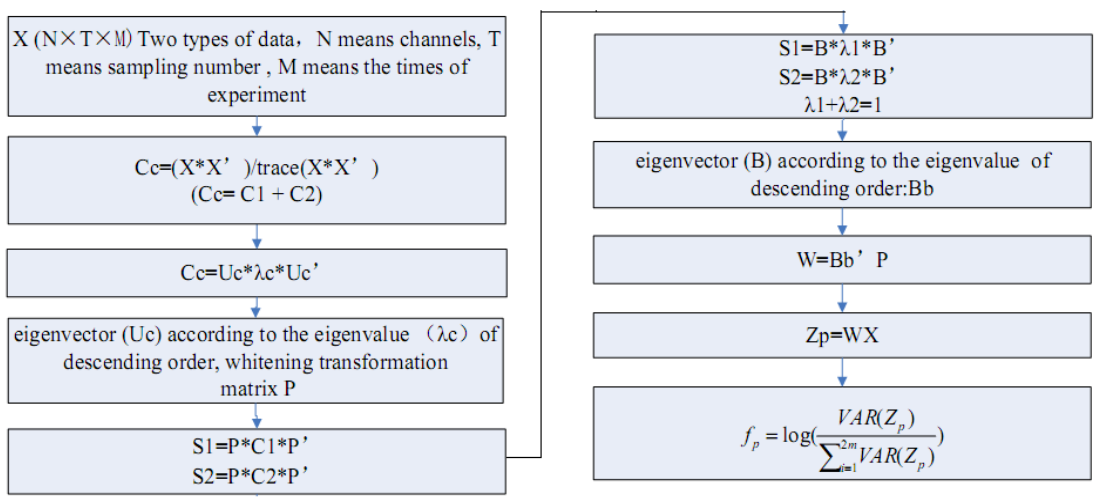

figure 3. CSP process

We can see the training data of two types go through the process in figure 3 , if choose $n=3$, can get the final train-data (100X6, 100 means the number of experiments, 6 means the number of feature values $)$. And test data $(32 \times 2000 \times 100)$ passes the spatial filter matrix, the final data is the test-data $(100 \times 6)$.

\section{Feature classification}

Support vector machine (SVM) is a machine learning method based on statistical learning theory. It through the proper nonlinear mapping (kernel functions) makes input vector mapped to a highdimensional feature space, in order to make the data (two classes) be divided completely [7]. EEG signal has nonlinear characteristics. For the nonlinear classification problem, Training set through a nonlinear function $\mathrm{x}$, maps to a high dimensional linear feature space, and the linear feature space constructs a hyper plane classifier. The optimal classification surface would require classifier not only can separate two classes of samples without error, but also make the gap (Margin) of the two types as large as possible [8].

The main idea of SVM is to set up a hyper plane as surface of decision-making, makes the distance between sample data and the hyper plane as large as possible. Then the hyper plane is shown in figure 4 .

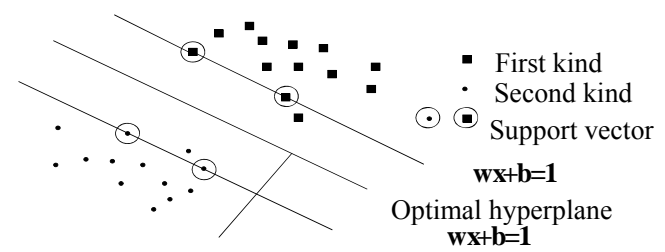

Original point $w x+b=1$

Figure 4.Optimal hyper plane

Radial basis kernel function is commonly used kernel function of SVM, with few parameters, low model complexity. We choose equation (1) as the kernel function of SVM for the nonlinear mapping conversion of low dimensional space to high dimension space [9].

$$
k\left(x_{i}, x_{j}\right)=\exp \left(-\gamma\left\|x_{i}-x_{j}\right\|^{2}\right), \gamma>0
$$

Where xi means the first $\mathrm{i}$ input samples, $\gamma$ nuclear parameter.

This paper adopts LIBSVM which developed by National Taiwan University professor Lin. LIBSVM provides a parameter selection tool for the radial basis kernelcross validation via parallel grid search[4]. In the process of classification, firstly, we can get the model of training data. On the basis of the training data model, we can get the feature values of test data, and then predict the final train-data $(100 \times 6,100$ means the number of experiments, 6 labels of the test data). And the accuracy can be calculated by comparing the actual labels and the predicted labels. 
Table 1 Classification accuracy of several subjects

\begin{tabular}{|c|c|c|c|}
\hline Numble & Filter settings & $\begin{array}{c}\text { SVM parameters } \\
(-\mathrm{c}, \mathrm{g})\end{array}$ & classification rate(\%) \\
\hline M1 & $(5-30) \mathrm{Hz}$ & $\mathrm{c}=1024, \mathrm{~g}=0.5$ & 86.67 \\
\hline M2 & $(5-30) \mathrm{Hz}$ & $\mathrm{c}=1024, \mathrm{~g}=87.5$ & 87.50 \\
\hline M3 & $(5-30) \mathrm{Hz}$ & $\mathrm{c}=1024, \mathrm{~g}=128$ & 83.50 \\
\hline F1 & $(5-30) \mathrm{Hz}$ & $\mathrm{c}=1024, \mathrm{~g}=0.25$ & 85.00 \\
F2 & $(5-30) \mathrm{Hz}$ & $\mathrm{c}=16, \mathrm{~g}=4$ & 89.20 \\
F3 & $(5-30) \mathrm{Hz}$ & $\mathrm{c}=1024, \mathrm{~g}=0.015$ & \\
\hline
\end{tabular}

\section{Analysis of the emotions based on wavelet entropy}

Wavelet transform and wavelet entropy have important application in the characteristic study of imaginative movement electroencephalogram signal change.

Supposing signal was decomposed by multi-scale wavelet on the M scale. The wavelet coefficient vector on scale $\mathrm{j}$ is $\mathrm{W}_{-} \mathrm{j}=\left(\mathrm{w}_{-} \mathrm{j} 1, \mathrm{w}_{-} \mathrm{j} 2, \ldots, \mathrm{w}_{-} \mathrm{jn}\right)$. The energy of the scale $\mathrm{J}$ is defined as [10]:

$$
E_{j}=\left\|W_{j}\right\|^{2}=\sum_{i=1}^{n}\left|w_{j i}\right|^{2}
$$

The distribution of energy sequence is defined as the normalized energy of each scale:

The total wavelet energy is $\mathrm{E}=\sum_{j=1}^{M} E_{j}$.

$$
p_{i}=E_{i} / E \quad \mathrm{i}=1,2, \ldots, \mathrm{M}
$$

Combined with the definition of information entropy. Energy sequence distribution in various scales of wavelet $P$ replace the probability distribution of signal. Entropy based on energy distribution is called wavelet entropy [11].

$$
H_{w e}=H(P)=H\left(p_{1}, p_{2}, \ldots, p_{m}\right)=\sum_{i=1}^{m} p_{i} \log _{2} p_{i}
$$
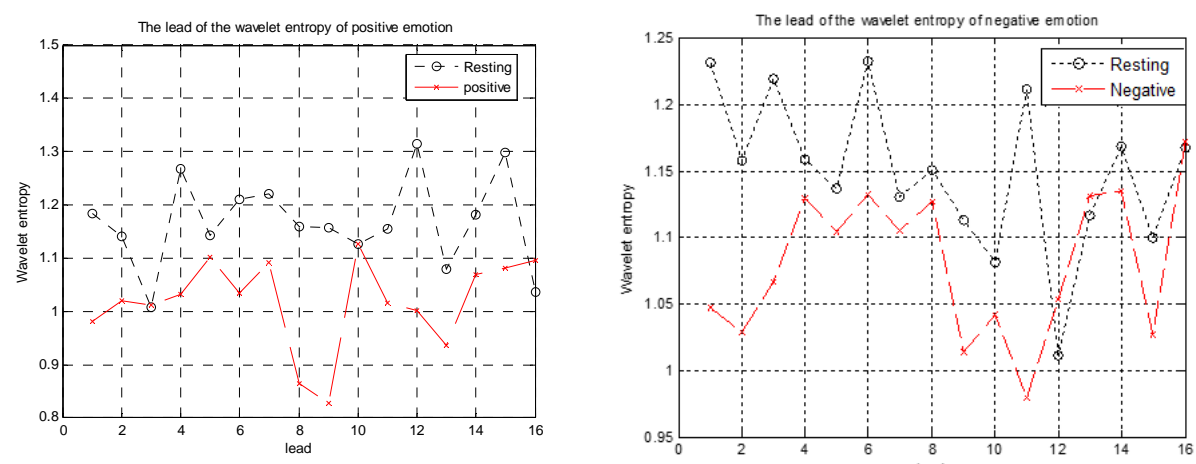

Figure5. Analysis of the emotions based on wavelet entropy

We can see from the picture that leads which related to the emotion mainly concentrated in the areas of FP, FP2, O1, F7, F8, T5. In order to verify the difference of positive emotion and negative emotion, we also analyzed the wavelet entropy of two emotions. As shown in the following figure.

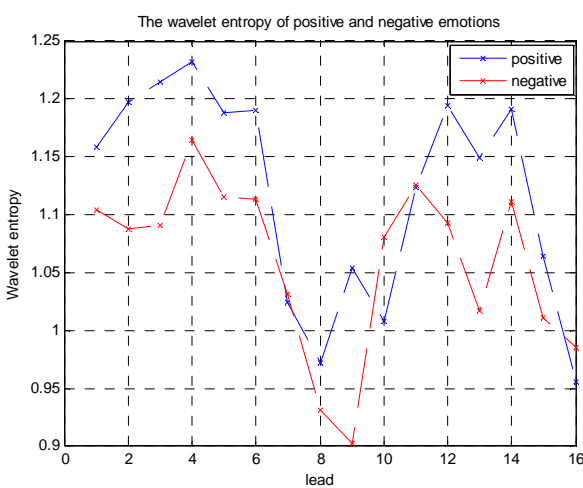

Figure6. The wavelet entropy of positive and negative emotions 
It found that wavelet entropy value of the negative emotion state is lower than the positive state. It shows that the brain is nervous and regularity of the brain is stronger when it is negative. In the positive mood, however, the brain is relatively relaxed and the regularity becomes weak.

\section{Summary}

In this paper, we analyzed the difference of positive and negative emotions based on EEG signals. Two kinds of signals are successfully classified. By using wavelet entropy to analyze two kinds of EEG signals of different emotions and the result is obvious.

This study provides great value for the study of depression and those who can be easily influenced by emotion for heavy stress.

\section{References}

[1] Blanchette I, Richards A, The influence of effect on higher level cognition: A review of research on interpretation, judgement, decision making and reasoning, Cognition\&Emotion,2010, 24(4): 561-595

[2] Frantzidis C A, Bratsas C, Klados M A, et al, On the classification of emotional biosignalsevoked while viewing affective pictures: an integrated data-mining based approach for healthcare applications, Information Technology in Biomedicine, 2010, 14(2): 309-318

[3] P.Ekman, Basic Emotions, in Handbook of cognition and emotion, T.Dagleish and M.Power, Eds, Sussex, U.K.:John Wiiey\&Sons Ltd.1999,45-60

[4] Russell JA. A circumplex model of affect [J]. J Pers SocPsychol 1980; 39(6):116121178

[5] Lang PJ. The emotion probe. Studies of motivation and attention [J]. Am Psycho1, 1995, 50(5):372-385

[6] H.Ramoser, J.M• uler-Gerking, G.Pfurtscheler. Optimal spatial filtering of single trial during imagined hand movement EEG. IEEE TRANSACTIONS ONREHABILITATION ENGINEER ING, VOL.XX, NO.Y, AUG.1998.

[7] Chong L. Classification of motor imagery EEG signals based on CSP and SVM algorithm [J]. Journal of Northeastern University,2010; 31(8):1098-1101

[8] Shengdong D, Support vector machine method in pattern recognition[D].Chongqing: Master's degree thesis of Chongqing University,2007

[9] Mingai L , Motor imagery EEG signal recognition method based on improved CSP algorithm Biomedical Engineering.2009.28(2):161-165

[10] Svaldo A Rosso, Susana Blanco,et al.Waveleten-tropy:a new tool for analysis of short doration brain electrical signals.Journal of Neuroscience Methods.2001;105:65-75.

[11]Xiaochen B. MATLAB toolbox application guide.Beijing: Publishing House of Electronics Industry.2000:307-397 